\title{
Under pressure: quenching star formation in low-mass satellite galaxies via stripping
}

\author{
Sean P. Fillingham, ${ }^{1 \star}$ Michael C. Cooper, ${ }^{1 \star}$ Andrew B. Pace, ${ }^{1}$ \\ Michael Boylan-Kolchin, ${ }^{2}$ James S. Bullock, ${ }^{1}$ Shea Garrison-Kimmel ${ }^{3} \dagger$ \\ and Coral Wheeler ${ }^{1}$ \\ ${ }^{1}$ Center for Cosmology, Department of Physics and Astronomy, 4129 Reines Hall, University of California, Irvine, CA 92697, USA \\ ${ }^{2}$ Department of Astronomy, The University of Texas at Austin, 2515 Speedway, Stop C1400, Austin, TX 78712, USA \\ ${ }^{3}$ TAPIR, Mailcode 350-17, California Institute of Technology, Pasadena, CA 91125, USA
}

Accepted 2016 August 22. Received 2016 August 18; in original form 2016 June 21

\begin{abstract}
Recent studies of galaxies in the local Universe, including those in the Local Group, find that the efficiency of environmental (or satellite) quenching increases dramatically at satellite stellar masses below $\sim 10^{8} \mathrm{M} \odot$. This suggest a physical scale where quenching transitions from a slow 'starvation' mode to a rapid 'stripping' mode at low masses. We investigate the plausibility of this scenario using observed $\mathrm{H}_{\text {I }}$ surface density profiles for a sample of 66 nearby galaxies as inputs to analytic calculations of ram-pressure and turbulent viscous stripping. Across a broad range of host properties, we find that stripping becomes increasingly effective at $M_{*} \lesssim 10^{8-9} \mathrm{M}_{\odot}$, reproducing the critical mass scale observed. However, for canonical values of the circumgalactic medium density $\left(n_{\text {halo }}<10^{-3.5} \mathrm{~cm}^{-3}\right)$, we find that stripping is not fully effective; infalling satellites are, on average, stripped of only $\lesssim 40-60$ per cent of their cold gas reservoir, which is insufficient to match observations. By including a host halo gas distribution that is clumpy and therefore contains regions of higher density, we are able to reproduce the observed $\mathrm{H}_{\text {I }}$ gas fractions (and thus the high quenched fraction and short quenching timescale) of Local Group satellites, suggesting that a host halo with clumpy gas may be crucial for quenching low-mass systems in Local Group-like (and more massive) host haloes.
\end{abstract}

Key words: galaxies: dwarf-galaxies: evolution-galaxies: formation-galaxies: generalLocal Group-galaxies: star formation.

\section{INTRODUCTION}

Recent studies probing the properties of satellite galaxies in the local Universe show that the suppression (or 'quenching') of star formation in satellites is a relatively inefficient process relative to the expectations of hydrodynamic and semi-analytic models of galaxy formation (e.g. Kimm et al. 2009; Kimm, Yi \& Khochfar 2011; Hirschmann et al. 2014; Wang et al. 2014; Phillips et al. 2015). While satellites are rapidly quenched - following infall - in the models, analysis of satellite populations identified in the Sloan Digital Sky Survey (SDSS; York et al. 2000) instead find that quenching proceeds remarkably slowly, such that a typical satellite with $M_{\star}$ $\gtrsim 10^{8} \mathrm{M}_{\odot}$ orbits within its host halo - continuing to form stars - for 3-7 Gyr before being quenched (De Lucia et al. 2012; Wetzel et al. 2013; Wheeler et al. 2014). ${ }^{1}$ Only at the lowest satel-

\footnotetext{
^E-mail: sfilling@uci.edu (SPF); cooper@uci.edu (MCC)

$\dagger$ Einstein Fellow.

${ }^{1}$ These measured satellite quenching time-scales include the transition of a satellite system from star forming to quiescent, which must proceed quickly
}

lite masses is quenching a highly efficient process, with low-mass $\left(M_{\star}<10^{8} \mathrm{M}_{\odot}\right)$ satellites in the Local Group quenching within $\sim 1-$ 2 Gyr of infall (Fillingham et al. 2015; Weisz et al. 2015; Wetzel, Tollerud \& Weisz 2015b).

In an effort to connect these measured quenching time-scales to the relevant physical mechanisms at play, Fillingham et al. (2015) present a comprehensive picture of satellite quenching spanning roughly 5 orders of magnitude in satellite stellar mass. The low efficiency and long quenching time-scales inferred for intermediateand high-mass satellites $\left(M_{\star} \gtrsim 10^{8} \mathrm{M}_{\odot}\right)$ are consistent with quenching via starvation - a scenario in which gas accretion on to a satellite galaxy is halted following infall, thus eventually eliminating the fuel for star formation (Larson, Tinsley \& Caldwell 1980; Kawata \& Mulchaey 2008); lending support to this picture, the measured quenching time-scales agree very well with the observed cold gas $\left(\mathrm{H}_{\mathrm{I}}+\mathrm{H}_{2}\right)$ depletion time-scales for field systems at $z \sim 0$

(within $\sim 1 \mathrm{Gyr}$ ) so as to reproduce the observed bimodal distribution of specific star formation rates and rest-frame colours (Balogh et al. 2004; Wetzel et al. 2013). 
(Fillingham et al. 2015). At lower satellite masses $\left(M_{\star} \lesssim 10^{8} \mathrm{M}_{\odot}\right)$, however, the quenching time-scales derived from analysis of the Local Group satellite population suggest that the physics of satellite quenching must change significantly; a more efficient quenching mechanism (relative to starvation) must be at play below a critical mass scale of $\sim 10^{8} \mathrm{M}_{\odot}$.

Stripping is a plausible candidate quenching mechanism at low masses. This includes ram-pressure stripping (Gunn \& Gott 1972), a process by which the cool, dense interstellar medium (and thus the fuel for future star formation) is removed from a satellite galaxy as it passes through its host's circumgalactic medium (CGM). Rampressure stripping becomes increasingly effective in lower mass satellites, due to their weaker gravitational restoring pressures (Hester 2006); moreover, ram pressure acts on roughly the dynamical time of the host system (i.e. 1-3 Gyr; Tonnesen, Bryan \& van Gorkom 2007; Bekki 2014), consistent with the short quenching time-scale inferred for low-mass satellites of the Local Group. In addition to ram-pressure stripping, cold gas may also be removed from a satellite due to turbulent viscous stripping, which results from Kelvin-Helmholtz instabilities at the interface of the satellite's interstellar medium and the CGM (Nulsen 1982). The relative motion of the two media in addition to the substantial difference in their mean densities can lead to perturbations that overcome the local gravitational restoring force, such that gas is stripped from the satellite. Within massive groups and clusters, a wide range of observations provide abundant evidence of stripping in action, showing its ability to quench infalling galaxies via removal of their cold gas component (e.g. Ebeling, Stephenson \& Edge 2014; Kenney, Abramson \& Bravo-Alfaro 2015). It remains uncertain, however, if stripping is an effective quenching mechanism in more typical host haloes, such as that of the Milky Way or M31, and specifically at satellite stellar masses of $\lesssim 10^{8} \mathrm{M}_{\odot}$.

In this work, our goal is to directly address the efficacy of stripping as a quenching mechanism for low-mass satellites in Milky Waylike systems. By using observations of local field dwarfs to inform analytic calculations of both ram-pressure and turbulent viscous stripping, we measure the amount of cold gas that would typically be removed if these field dwarfs were to interact with a Milky Waylike host. In Section 2, we detail our methods, including the analytic framework and data sets that we utilize to estimate the impact of stripping on infalling satellites. In Section 3, we present our primary results regarding the efficiency of stripping in Milky Waylike environments, specifically addressing potential uncertainties associated with the properties of the host halo and the satellite population. Finally, in Sections 4 and 5, we discuss and summarize the implications of our results with regard to the quenching of lowmass satellites in the Local Group and beyond.

\section{TESTING SATELLITE STRIPPING}

\subsection{Analytic framework}

The effectiveness of stripping as a quenching mechanism boils down to a relatively simple competition between the stripping pressure $\left(P_{\text {stripping }}\right)$ and the gravitational restoring force per unit area (i.e. the gravitational restoring pressure, $P_{\text {restore }}$ ). When $P_{\text {restore }} \geq$ $P_{\text {stripping }}$, the interstellar medium (or ISM, comprised predominantly of cold gas) is retained by the infalling satellite galaxy, such that star formation may proceed. When the stripping pressure exceeds the gravitational restoring pressure, however, some fraction of the cold gas is removed from the satellite. In cases where this stripped fraction is large enough, star formation will be shut down rapidly due to the loss of available fuel. In what follows, we investigate two different mechanisms for removing the cold interstellar medium of infalling satellites.

\subsubsection{Ram-pressure stripping}

Following Gunn \& Gott (1972), we estimate the ram pressure $\left(P_{\text {ram }}\right)$ as:

$P_{\text {ram }} \sim \rho_{\text {halo }} V_{\text {sat }}^{2}$,

where $\rho_{\text {halo }}$ is the density of the host's gas halo and $V_{\text {sat }}$ is the velocity of the satellite galaxy with respect to the host's frame of reference, or more precisely the local reference frame of the host's gas halo in the immediate vicinity of the infalling satellite galaxy. As shown in equation (1), the ram pressure experienced by an infalling satellite is dependent on the local environment - thus, the properties of the host system, in particular its dark matter halo mass, which plays a critical role in setting $\rho_{\text {halo }}$ and $V_{\text {sat }}$. As described in Section 2.2, we utilize $N$-body simulations and observations of the Local Group and similar systems to inform our selection of these global environmental parameters, applying average values of $\rho_{\text {halo }}$ and $V_{\text {sat }}$ to all infalling satellites in our analysis. The adopted values for these parameters, along with uncertainties or biases that their selection introduces, are discussed directly in Section 3.2.

Assuming a spherical mass profile for an infalling satellite, the gravitational restoring force per unit area is given by:

$P_{\text {restore }} \sim \Sigma_{\text {gas }}(r) \frac{G M(r)}{r^{2}}$,

where $\Sigma_{\text {gas }}(r)$ is the surface density of the cold gas to be stripped from the satellite and $M(r)$ is the total satellite mass interior to the radius $r$. As shown in equation (2), the restoring pressure depends exclusively on the properties of the infalling galaxy, varying from one satellite system to the next, as it is accreted on to the parent halo. To model the properties of a representative sample of infalling satellites, we utilize observational data for a broad collection of nearby galaxies, including mass modelling to infer the local gravitational potential on a system-by-system basis (see Section 2.3).

For an infalling satellite, the degree to which ram pressure is able to strip its ISM is determined by the relative magnitude of the two pressures ( $P_{\text {ram }}$ versus $\left.P_{\text {restore }}\right)$, such that stripping will occur beyond a radius $r$ (within the satellite) if

$\rho_{\text {halo }} V_{\text {sat }}^{2}>\Sigma_{\text {gas }}(r) \frac{G M(r)}{r^{2}}$.

Throughout this work, we define $R_{\text {strip }}$ as the innermost radial distance at which this inequality holds. Inside $R_{\text {strip }}$, the restoring pressure is able to resist stripping, while ram pressure dominates beyond this radius.

\subsubsection{Turbulent viscous stripping}

The interaction at the interface of the ISM and the CGM can result in the growth of Kelvin-Helmholtz $(\mathrm{K}-\mathrm{H})$ instabilities due to the relative motion between the two phases. This will allow turbulent viscous stripping to remove the outer regions of the ISM when the gravitational restoring force is sufficiently small. Perturbations with wavenumber, $k$, are unstable if they meet the following criteria (Murray et al. 1993; Mori \& Burkert 2000):

$k>g \frac{\rho_{\text {gas }}^{2}-\rho_{\text {halo }}^{2}}{\rho_{\text {gas }} \rho_{\text {halo }} V_{\text {sat }}^{2}}$,

where $g$ is the gravitational restoring force at the ISM-CGM interface. 
Previous studies of turbulent viscous stripping find that the dominant wavelength is set by the size of the cold gas region $\left(R_{\mathrm{ISM}}\right)$, such that $k=2 \pi / R_{\mathrm{ISM}}$ (Nulsen 1982; Murray et al. 1993). In our analysis, we make the assumption that $\rho_{\text {halo }} \ll \rho_{\text {gas }}$, which is undoubtedly true for gas-rich dwarfs accreted into the Local Group (or similar environments). Plugging these approximations into equation (4), leads to the following inequality of the same form as equation (3):

$\rho_{\text {halo }} V_{\text {sat }}^{2}>\frac{G M_{0} \bar{\rho}_{\text {gas }}}{2 \pi R_{\mathrm{ISM}}}$,

where $\bar{\rho}_{\text {gas }}$ is the average ISM density inside $R_{\mathrm{ISM}}$, and $M_{0}$ is the total restoring mass inside $R_{\mathrm{ISM}}$. If this inequality is true, then turbulent viscous stripping will proceed and the outer layers of the ISM will be removed. When this inequality is false, the gravitational restoring pressure is able to stabilize the outer layers of the ISM against the $\mathrm{K}-\mathrm{H}$ instabilities.

When turbulent viscous stripping is able to proceed, the rate at which the ISM is removed will determine how much gas is stripped and ultimately whether the reservoir for star formation will be significantly depleted. The rate of total gas mass-loss $(\dot{M})$ is given in slightly different forms throughout the literature (e.g. Nulsen 1982; Mori \& Burkert 2000; Roediger \& Hensler 2005). In this work, we adopt the following approximation from Roediger \& Hensler (2005):

$\dot{M} \approx 20\left(\frac{R_{\mathrm{ISM}}}{20 \mathrm{kpc}}\right)^{2}\left(\frac{n_{\text {halo }}}{10^{-3} \mathrm{~cm}^{-3}}\right)\left(\frac{V_{\text {sat }}}{1000 \mathrm{~km} \mathrm{~s}^{-1}}\right) \frac{\mathrm{M}_{\odot}}{\mathrm{yr}}$.

The details regarding how equations (5) and (6) are used to determine the fraction of ISM removed from an infalling dwarf galaxy are discussed further in Section 2.4.

\subsection{Estimating $\rho_{\text {halo }}$ and $V_{\text {sat }}$}

The strength of the stripping force acting upon an infalling satellite is primarily set by the density of the host's halo gas along with the relative velocity of the satellite (see equations 1 and 5). Within the Milky Way, a variety of indirect probes point towards halo gas densities of $\sim 10^{-4} \mathrm{~cm}^{-3}$ for the hot $\left(T \sim 10^{6} \mathrm{~K}\right)$ component (e.g. Weiner \& Williams 1996; Stanimirović et al. 2002; Fox et al. 2005; Grcevich \& Putman 2009; Salem et al. 2015). Moreover, both observed $\mathrm{X}$-ray emission and pulsar dispersion measurements in the Milky Way are consistent with a cored hot halo distribution with a density of $>10^{-4} \mathrm{~cm}^{-3}$ extending to radial distances of $\sim 100 \mathrm{kpc}$ (Fang, Bullock \& Boylan-Kolchin 2013, see also Anderson \& Bregman 2010; Gupta et al. 2012; Miller \& Bregman 2013, 2015; Faerman, Sternberg \& McKee 2016).

While the Milky Way's hot halo component is clearly important with regard to stripping in the Local Group, it is the density of the host's halo gas - across all temperatures - that dictates the strength of the stripping force. When folding in cooler phases of the CGM, recent studies of nearby massive galaxies, comparable to the Milky Way, find halo gas densities of $\sim 10^{-3.5} \mathrm{~cm}^{-3}$ extending to at least $\gtrsim 0.25 R_{\text {vir }}$ (Tumlinson et al. 2013; Werk et al. 2014; Faerman et al. 2016). These results are also supported by the latest analysis of the CGM surrounding M31 using quasar absorptionline spectroscopy, which finds evidence for a massive and extended gas halo (Lehner, Howk \& Wakker 2015). Related studies targeting more massive, high- $z$ systems find extended, high-density reservoirs of cool $\left(10^{4} \mathrm{~K}\right)$ halo gas reaching out to large fractions of the virial radius (Lau, Prochaska \& Hennawi 2015). In accordance with these recent results, we assume a fiducial value for the host halo density $\left(n_{\text {halo }}\right)$ of $10^{-3.5} \mathrm{~cm}^{-3}$, where $\rho_{\text {halo }}=\mu n_{\text {halo }} m_{\mathrm{HI}}$. For the purposes of this analysis, the mean molecular weight, $\mu$, is set to 1 .

Given the significant uncertainties in the observed halo densities of the Milky Way and M31, we explore how both our calculations of instantaneous ram-pressure stripping and continuous viscous stripping depend on this adopted value of $n_{\text {halo }}$ in Section 3.2. Throughout our analysis, we make no assumptions regarding the radial profile of the halo gas. Given the expected orbits of infalling satellite populations and the existing constraints on the quenching time-scale measured relative to infall, however, there are relevant constraints regarding the extent of the CGM, which we discuss in Section 4.4.

To estimate the relative velocity of a satellite system in relation to the host's halo gas $\left(V_{\text {sat }}\right)$, we study the distribution of subhalo velocities within the Exploring the Local Volume In Simulations (ELVIS) suite of 48 high-resolution, dissipationless simulations of Milky Way-like haloes (Garrison-Kimmel, Boylan-Kolchin, Bullock \& Lee 2014a). The ELVIS suite includes 24 isolated haloes as well as 12 mass-matched Local Group-like pairs, simulated within highresolution uncontaminated volumes spanning 2-5 Mpc in size using a particle mass of $1.9 \times 10^{5} \mathrm{M}_{\odot}$ and a Plummer-equivalent force softening of $\epsilon=141$ physical parsecs. Within the high-resolution volumes, the halo catalogs are complete down to $M_{\text {halo }}>2 \times$ $10^{7} \mathrm{M}_{\odot}, V_{\max }>8 \mathrm{~km} \mathrm{~s}^{-1}, M_{\text {peak }}>6 \times 10^{7} \mathrm{M}_{\odot}$, and $V_{\text {peak }}>$ $12 \mathrm{~km} \mathrm{~s}^{-1}$ - thus more than sufficient to track the evolution of haloes hosting the Local Group dwarf population.

From ELVIS, we select subhaloes corresponding to satellites with stellar masses of $10^{6}-10^{9} \mathrm{M}_{\odot}$ - i.e. halo masses of $10^{9.7}-10^{11.2} \mathrm{M}_{\odot}$ following the stellar mass-halo mass (SMHM) relation of GarrisonKimmel et al. (2014a). We sample the velocities of these subhaloes at the time of infall (i.e. crossing $R_{\mathrm{vir}}$ ), at $0.5 R_{\mathrm{vir}}$, and at pericentre. In Fig. 1, we show the distribution of subhalo velocities (relative to

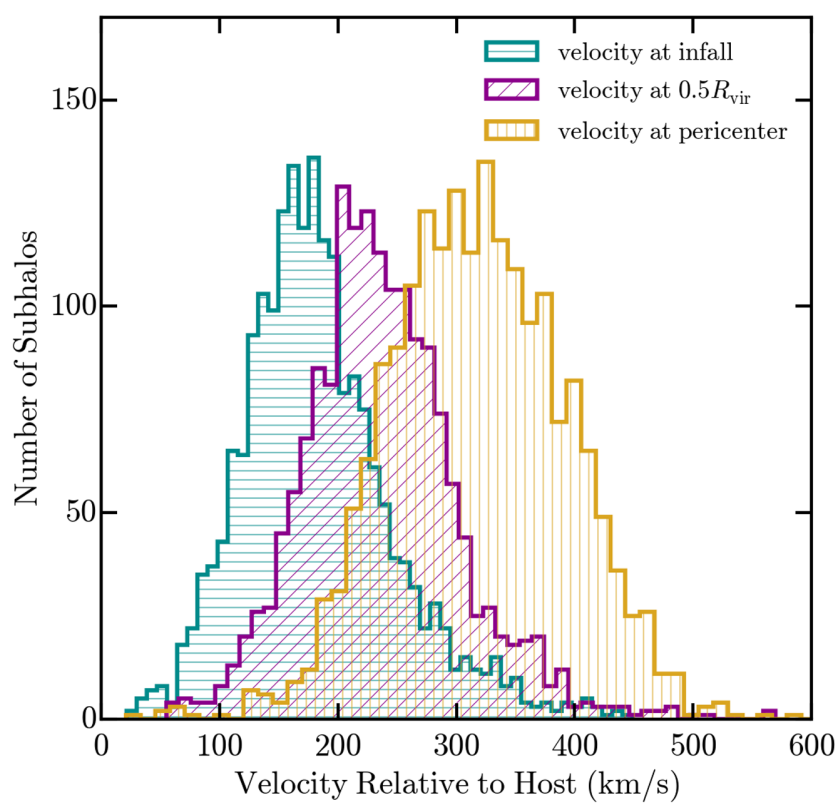

Figure 1. The distribution of subhalo velocities (relative to their parent halo) in the ELVIS suite of Local Group simulations for all subhaloes that reside inside the virial radius at $z=0$. The cyan, magenta, and gold histograms correspond to subhalo velocities measured when crossing the virial radius (i.e. infall), at $0.5 R_{\text {vir }}$, and at pericentre, respectively. For Milky Way-like systems, the typical satellite velocity (relative to the host's halo gas) is roughly $200-400 \mathrm{~km} \mathrm{~s}^{-1}$ at the time of quenching. In our analysis, we adopt a fiducial value of $V_{\text {sat }}=300 \mathrm{~km} \mathrm{~s}^{-1}$. 
that of their host dark matter halo) at each of these distances. As expected, the average velocity of the subhalo population increases from infall towards pericentre, with a mean velocity of 183,237 , and $318 \mathrm{~km} \mathrm{~s}^{-1}$ at $R_{\mathrm{vir}}, 0.5 R_{\mathrm{vir}}$, and $R_{\text {peri }}$, respectively. ${ }^{2}$ To increase the precision at which we are able to measure the position of pericentric passage and the velocity at pericentre for each subhalo, we 3 - $\mathrm{d}$ spline interpolate the position and velocity information for all subhaloes across the 75 simulation snapshots in ELVIS. While this interpolation scheme achieves a time resolution of $\sim 20 \mathrm{Myr}$, our resulting measurements of $R_{\text {peri }}$ and $V_{\text {peri }}$ are likely somewhat overand under-estimated, respectively.

Typically, studies of instantaneous ram-pressure stripping assume the satellite velocity at pericentre, where stripping is expected to be greatest. Quenching (i.e. stripping) when a satellite reaches a radial distance of $\sim 0.5 R_{\mathrm{vir}}$ while on first infall, however, is consistent with the inferred quenching time-scales for the Local Group satellite population (Fillingham et al. 2015). Herein, we compromise between these two scenarios, adopting a fiducial satellite velocity $\left(V_{\text {sat }}\right)$ of $300 \mathrm{~km} \mathrm{~s}^{-1}$. In Section 3.2, we explore how our results depend upon this choice of $V_{\text {sat }}$.

\subsection{Estimating $\Sigma_{\text {gas }}(r)$ and $M(r)$}

As shown in equation (2), the gravitational restoring pressure is dependent upon the properties of the infalling satellite, specifically the gas surface density and total mass profiles. To estimate these parameters for a representative sample of infalling satellites, we utilize existing observations of 66 nearby, star-forming dwarf galaxies from the THINGS, Little THINGS, and SHIELD data sets (Walter et al. 2008; Hunter et al. 2012; Cannon et al. 2011; McNichols et al., in preparation; Teich et al., in preparation). This sample is dominated by isolated (or 'field') systems, for which the ISM is largely unaltered by previous interactions with a more massive host system - i.e. ideal candidates to test the effectiveness of ram-pressure stripping as a quenching mechanism. While the satellites of the Milky Way and M31 were primarily accreted at $z \sim 0.5-1$ (Fillingham et al. 2015; Wetzel, Deason \& Garrison-Kimmel 2015a), our sample of nearby galaxies is expected to be similar in cold gas (specifically $\mathrm{H}$ I) content to similar systems at intermediate redshift (Popping, Behroozi \& Peeples 2015; Somerville, Popping \& Trager 2015).

For galaxies drawn from Little THINGS and SHIELD, we infer the stellar mass of the system using the published $V$-band absolute magnitudes (Hunter et al. 2012; Haurberg et al. 2015) and assuming a mass-to-light ratio of unity, which is roughly consistent with the expectations for a $\sim 1$ Gyr-old simple stellar population following a Salpeter initial mass function (e.g. Maraston 1998). For those systems selected from THINGS, we utilize the stellar mass estimates of Leroy et al. (2008), which are derived from Spitzer $3.6 \mu \mathrm{m}$ imaging assuming a $K$-band mass-to-light ratio of 0.5 . As discussed further in Section 3.1, uncertainties in the measured stellar masses for our sample have little impact on the quantitative or qualitative results of our analysis. For the 12 galaxies in the SHIELD sample, the resulting stellar mass estimates are in relatively good agreement with those derived from stellar population fits to multi-band Hubble Space Telescope photometry, with a typical offset (to higher masses) of 0.37 dex (McQuinn et al. 2015). Altogether, the sample of 66 field

\footnotetext{
${ }^{2}$ Both the subhalo velocity and pericentre distributions show no dependence on subhalo mass, consistent with the idea that the host potential is the primary driver of these subhalo properties.
}

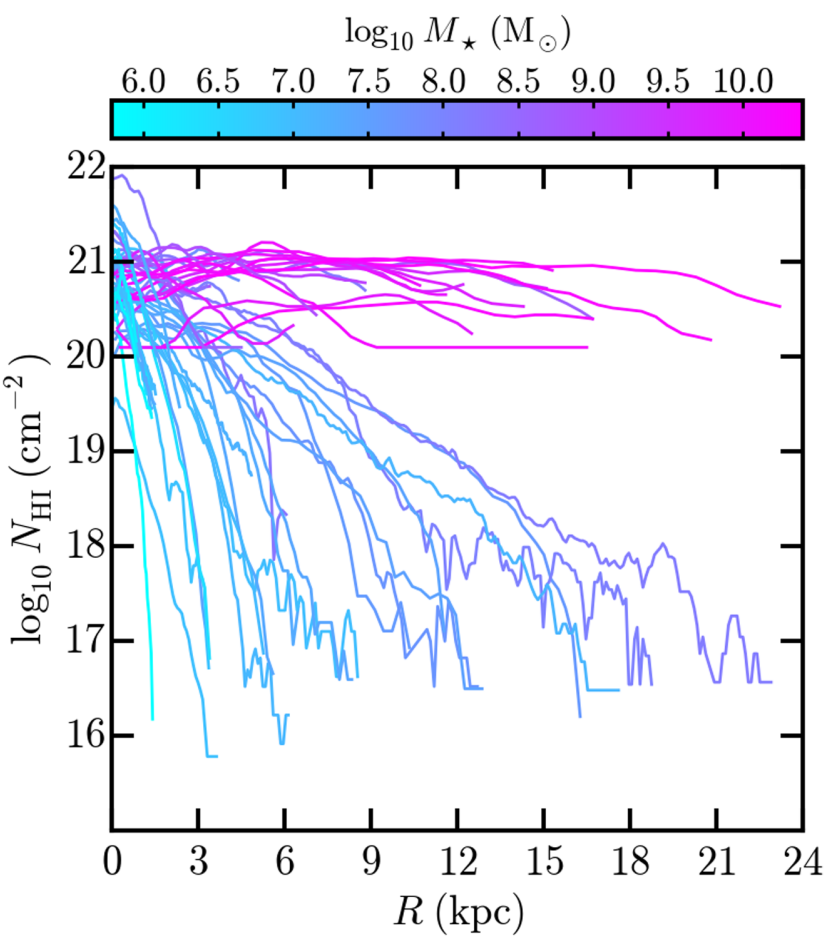

Figure 2. The observed H i surface density profiles for our sample of 66 nearby field galaxies from THINGS, Little THINGS, and SHIELD, colourcoded according to the stellar mass of each system. While there is substantial scatter in profile shape from object to object, the lower mass dwarfs are preferentially less extended than their more massive counterparts.

systems spans a broad range in stellar mass, from $\sim 10^{6}-10^{11} \mathrm{M}_{\odot}$, covering the mass regime where Fillingham et al. (2015) find evidence for a change in the dominant satellite quenching mechanism and where stripping is presumed to become effective.

For each of the galaxies in our sample, we utilize the published H I surface density profiles from the THINGS, Little THINGS, and SHIELD projects, scaled by a factor of 1.36 to account for helium (Leroy et al. 2008; Hunter et al. 2012; Teich, McNichols \& Cannon 2015; Teich et al., in preparation). For a typical low-mass galaxy, the cold gas component is largely dominated by atomic (versus molecular) gas (e.g. Boselli et al. 2014a; Popping, Somerville \& Trager 2014), such that $\Sigma_{\mathrm{H} \text { I }}$ provides a robust estimate of the ISM surface density and thus the efficacy of both ram-pressure and turbulent viscous stripping. As shown in Fig. 2, the $\Sigma_{\mathrm{HI}}$ profiles for our sample exhibit significant variation in shape, with more massive systems having preferentially more extended $\mathrm{H}_{\mathrm{I}}$ surface density profiles. While the depth of the $\mathrm{H}$ I observations varies from object to object in our sample, the THINGS, Little THINGS, and SHIELD measurements are sensitive to the bulk of the atomic gas component, such that any undetected low-density gas at large radii would have a negligible impact on our stripping calculations.

To determine the mass profile, $M(r)$, for each galaxy in our sample, we infer the total dark matter halo mass according to the stellar mass-halo mass relation of Garrison-Kimmel et al. (2014a) and assume an NFW density profile (Navarro, Frenk \& White 1997) with a concentration given by the $c-M$ relation of Klypin, TrujilloGomez \& Primack (2011). While this methodology neglects contributions to the mass profile from the baryonic component, these are relatively modest at these mass scales (i.e. $M_{\star}<10^{11} \mathrm{M}_{\odot}$ ), as illustrated in Section 3.1. Recognizing current uncertainties in the dark matter density profiles of low-mass galaxies (e.g. Moore 1994; 


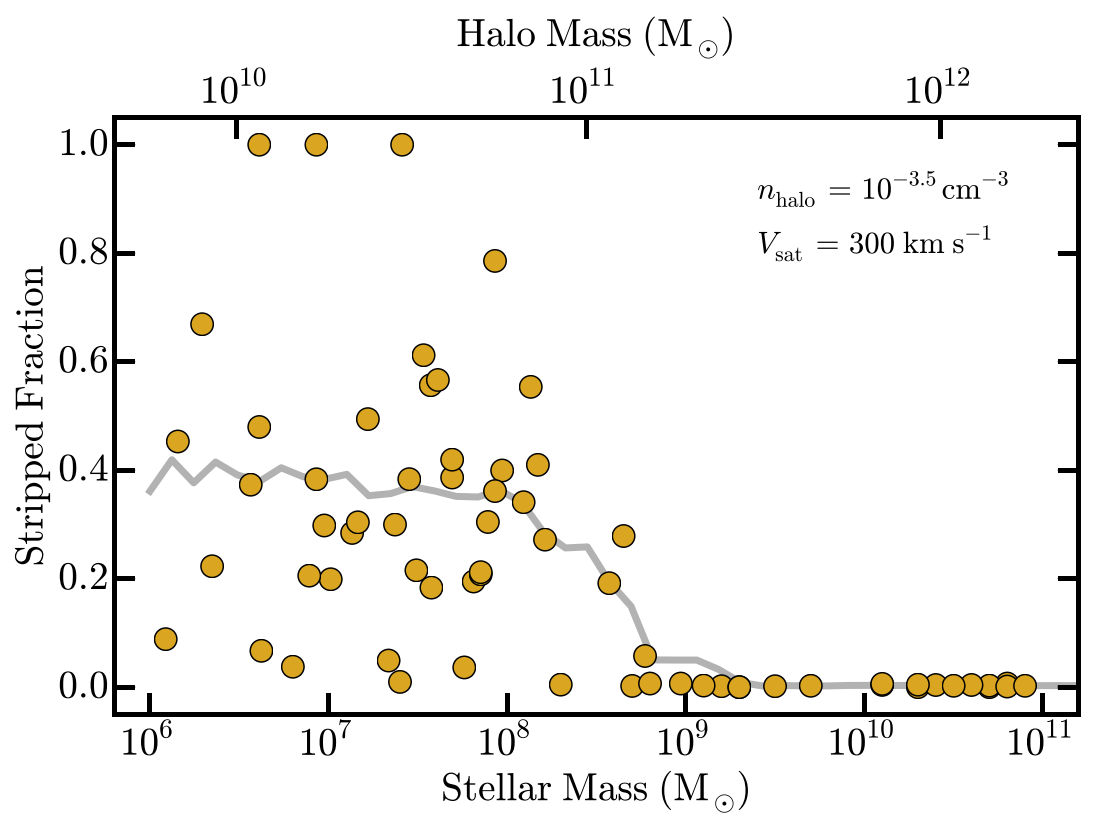

Figure 3. The fraction of $\mathrm{H}_{\mathrm{I}}$ gas stripped $\left(f_{\text {stripped }}\right)$ via ram pressure as a function of stellar mass for our sample of 66 dwarf galaxies, assuming a host halo gas density of $n_{\text {halo }}=10^{-3.5} \mathrm{~cm}^{-3}$ and a satellite velocity of $V_{\text {sat }}=300 \mathrm{~km} \mathrm{~s}^{-1}$. The grey solid line corresponds to the mean $f_{\text {stripped }}$ computed in a sliding bin of width 0.6 dex in stellar mass. At stellar masses greater than roughly $10^{9} \mathrm{M}_{\odot}$, we find that satellite systems are unaffected by ram pressure in a Milky Way-like environment. At $M_{\star} \lesssim 10^{8.5} \mathrm{M}_{\odot}$, however, ram pressure is increasingly effective, with infalling systems typically having $\sim 40$ per cent of their cold gas stripped. It is worth noting that the scatter in the stripped fraction at fixed stellar mass is driven entirely by the variation in the $\mathrm{H}$ I surface density profiles. For reference, we include the corresponding halo mass for each system, as inferred via the stellar mass-halo mass relation of Garrison-Kimmel et al. (2014a).

de Blok et al. 2001; Boylan-Kolchin, Bullock \& Kaplinghat 2011, 2012), we also employ mass profiles derived from dynamical modelling of the observed Hi kinematics for a subset of our systems, including NFW fits to the THINGS and Little THINGS samples from de Blok et al. (2008) and Oh et al. (2015) as well as fits to a Burkert profile (Burkert 1995) from Pace (2016).

\subsection{Measuring the stripped fraction $\left(f_{\text {stripped }}\right)$}

Using the H I surface density profiles presented in Fig. 2, we determine the fraction of $\mathrm{H}_{\mathrm{I}}$ gas stripped from each satellite in our sample, given an assumed host halo density $\left(\rho_{\text {halo }}\right)$, satellite velocity $\left(V_{\text {sat }}\right)$, and satellite mass profile $(M(r))$. Throughout our analysis, we first determine the amount of ISM removed via instantaneous ram-pressure stripping, then we allow turbulent viscous stripping to proceed for up to $1 \mathrm{Gyr}$.

\subsubsection{Ram-pressure stripping}

First, the satellite experiences ram pressure stripping, which is generally assumed to coincide with either initial infall or pericentric passage. As discussed in Section 2.1, equation (3) specifies the radial distance, measured from the centre of each satellite, at which ram pressure exceeds the gravitational restoring pressure $\left(R_{\text {strip }}\right)$. By integrating the $\mathrm{H}$ I surface density profile beyond this radius, we compute the fraction of gas stripped from each satellite as

$f_{\text {stripped }}=\frac{\int_{R_{\text {strip }}}^{R_{\max }} \Sigma_{\text {gas }}(r) r \mathrm{~d} r}{\int_{0}^{R_{\text {max }}} \Sigma_{\text {gas }}(r) r \mathrm{~d} r}$,

where $R_{\max }$ is the outermost radial distance at which $\mathrm{H}_{\mathrm{I}}$ is detected. Here, the numerator corresponds to the gas mass that is stripped from the satellite after it interacts with the CGM of the host. The denominator is the total gas mass that resides in the system in the absence of any environmental effects (i.e. prior to infall). The stripped fraction in this scenario is the amount of gas removed in a single, instantaneous interaction between the infalling satellite and the host halo.

As discussed in Section 2.3, the adopted definition for $R_{\max }$ leads to an underestimate of the stripped fraction, as low-density gas at large galactocentric radii is unaccounted for in our analysis. However, given the sensitivities of the THINGS, Little THINGS, and SHIELD H I maps, any $\mathrm{H}_{\mathrm{I}}$ component at large radii contributes minimally to the total atomic gas mass, such that the resulting impact on $f_{\text {stripped }}$ should be negligible.

\subsubsection{Turbulent viscous stripping}

After estimating $f_{\text {stripped }}$ as a result of ram-pressure stripping, we compute the corresponding fraction of gas removed due to turbulent viscous stripping over a maximum timespan of $1 \mathrm{Gyr}$. First, we test whether the ISM and CGM interface conditions located at $R_{\text {strip }}$ are susceptible to viscous stripping via equation (5). If true, we determine the gas mass lost during a $100 \mathrm{Myr}$ interval, $M_{\mathrm{viscous}}$, using equation (6). $M_{\text {viscous }}$ is then uniformly removed from the outermost regions of the $\mathrm{H}$ I surface density profile, leading to a new $R_{\text {strip }}$ in addition to a new value of both $\bar{\rho}_{\text {gas }}$ and $M_{0}$. The ISM-CGM conditions are then reevaluated allowing the gas removal process to continue if equation (5) is still true. We repeat this process for up to $1 \mathrm{Gyr}$, leading to a total gas mass lost via turbulent viscous stripping. This additional gas mass is added to the gas which was initially removed via ram pressure to get a total gas mass lost as a result of stripping.

We limit the timespan on which turbulent viscous stripping occurs, so as to roughly match the measured quenching time-scale 

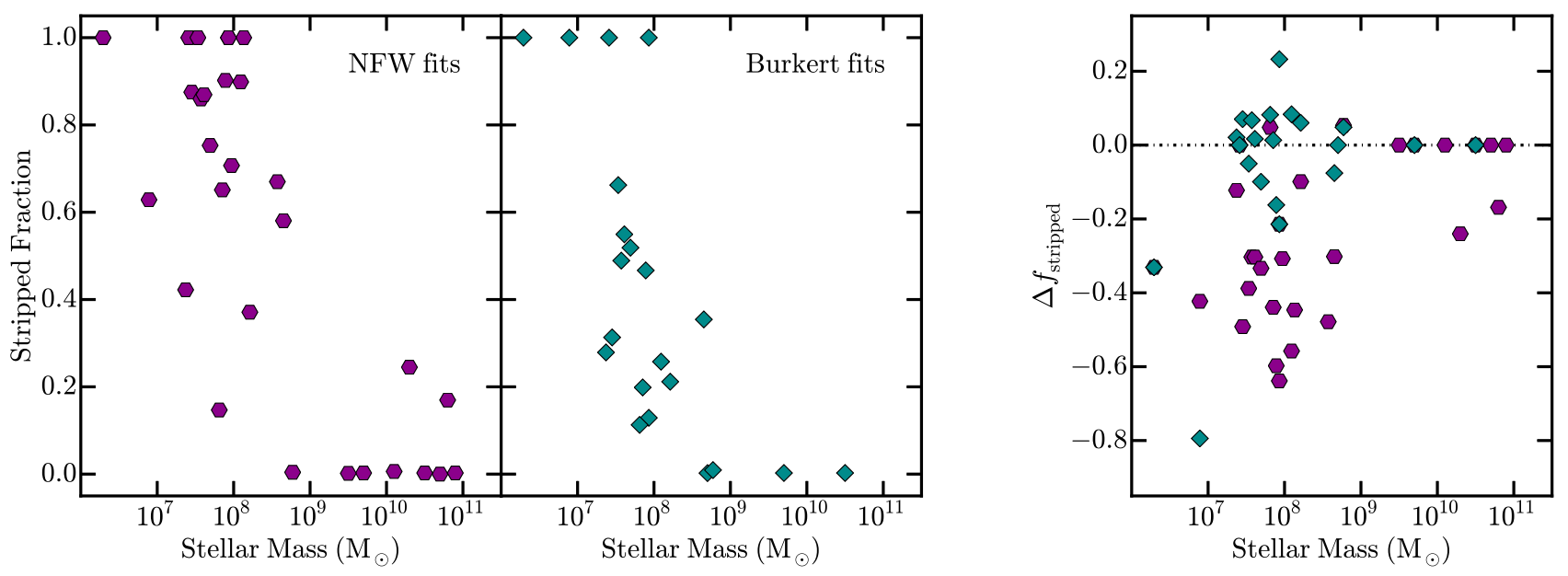

Figure 4. Left: the fraction of $\mathrm{H}$ g gas ram-pressure stripped $\left(f_{\text {stripped }}\right)$ as a function of stellar mass for the subset of systems with mass profiles determined via dynamical fits to an NFW profile (de Blok et al. 2008; Oh et al. 2015) and to a Burkert profile (Pace 2016). As in Fig. 3, we assume a halo gas density of $n_{\text {halo }}=$ $10^{-3.5} \mathrm{~cm}^{-3}$ and a satellite velocity of $V_{\mathrm{sat}}=300 \mathrm{~km} \mathrm{~s}^{-1}$. Right: the difference in the fraction of $\mathrm{H}_{\mathrm{I}}$ gas stripped relative to the corresponding result (see Fig. 3) assuming our fiducial mass profile inferred via the stellar mass-halo mass relation of Garrison-Kimmel et al. (2014a): $\Delta f_{\text {stripped }}=f_{\text {stripped, fid }}-f_{\text {stripped, dyn }}$. In general, the mass profiles inferred from dynamical modelling favour cored haloes, such that stripping is more efficient relative to our fiducial model. Moreover, while there is significant scatter from galaxy to galaxy based upon the assumed mass profile, the qualitative results are universal with ram-pressure stripping becoming increasingly effective at $M_{\star}<10^{8-9} \mathrm{M}_{\odot}$.

for Local Group satellites (i.e. $\lesssim 2$ Gyr; Fillingham et al. 2015). Approximately 50 percent (or 55 percent) of the subhalo population in ELVIS reaches pericentre (or $0.5 R_{\text {vir }}$ ) within $\sim 1.5 \mathrm{Gyr}$ (or $\sim 1 \mathrm{Gyr}$ ) of infall, where instantaneous ram-pressure stripping is assumed to occur. A further $1 \mathrm{Gyr}$ of turbulent viscous stripping therefore yields a typical quenching time (relative to infall) in rough agreement with the expectations of Fillingham et al. (2015) and Wetzel et al. (2015b).

\section{RESULTS}

In Fig. 3, we show the fraction of H I gas ram-pressure stripped from our sample of star-forming galaxies (with no viscous stripping), assuming a host halo density of $n_{\text {halo }}=10^{-3.5} \mathrm{~cm}^{-3}$ and a satellite velocity of $V_{\text {sat }}=300 \mathrm{~km} \mathrm{~s}^{-1}$. For satellite systems with stellar masses greater than $\sim 10^{9} \mathrm{M}_{\odot}$, ram pressure is unable to strip the interstellar medium, consistent with the long quenching time-scales inferred by Wheeler et al. (2014) and Fillingham et al. (2015) at this mass regime. At $M_{\star} \sim 10^{8-9} \mathrm{M}_{\odot}$, however, we find that ram pressure begins to overcome the local gravitational restoring force, such that a significant fraction of the cold gas reservoir is removed from a typical infalling satellite at $M_{\star}<10^{8} \mathrm{M}_{\odot}\left(<f_{\text {stripped }}\right.$ $>\sim 40$ per cent). While there is considerable scatter in the efficacy of ram-pressure stripping at low masses, our fiducial model for a Milky Way-like system qualitatively reproduces the critical mass scale for quenching at $\sim 10^{8} \mathrm{M}_{\odot}$, such that ram-pressure stripping is a viable candidate to be the dominant quenching mechanism at low satellite stellar masses. In Sections 3.1 and 3.2, we explore how this result depends on the specific parameters adopted in our fiducial model (i.e. $M(r), n_{\text {halo }}$, and $V_{\text {sat }}$ ). Additionally, in Section 3.3, we discuss how the inclusion of turbulent viscous stripping impacts these results.

\subsection{Dependence on $M(r)$}

The assumed dark matter density profile for each galaxy in our sample is critical in determining the strength of the local gravita- tional restoring pressure (see equation 2) and thus the degree to which ram pressure is able to strip the infalling satellite's ISM. In our fiducial model, the mass profiles, $M(r)$, are determined using the stellar mass-halo mass (SMHM) relation of Garrison-Kimmel et al. (2014a), assuming an NFW density profile. At low masses, however, less-cuspy dark matter profiles are typically favoured and there are large uncertainties in the slope of the stellar mass-halo mass relation (and its scatter, Garrison-Kimmel et al. 2016).

To explore how our estimates of $f_{\text {stripped }}$ depend on the assumed mass profile, $M(r)$, we utilize alternative mass profiles derived from dynamical fits to the observed $\mathrm{H}_{\mathrm{I}}$ kinematics for a subset of the systems in our sample. In particular, we utilize the NFW fits to the THINGS and Little THINGS velocity fields from de Blok et al. (2008) and Oh et al. (2015), respectively. For 21 galaxies, we also employ Burkert profile fits to the $\mathrm{H}$ i kinematics from Pace (2016).

The two left most panels of Fig. 4 show the fraction of atomic gas stripped for the subset of objects with dynamical mass estimates, assuming a host halo density of $n_{\text {halo }}=10^{-3.5} \mathrm{~cm}^{-3}$ and a satellite velocity of $V_{\text {sat }}=300 \mathrm{~km} \mathrm{~s}^{-1}$. Qualitatively, the dependence of $f_{\text {stripped }}$ on satellite stellar mass is very similar to that shown in Fig. 3 for our fiducial model, which employs mass profiles inferred from the SMHM relation of Garrison-Kimmel et al. (2014a). At $M_{\star}$ $<10^{8-9} \mathrm{M}_{\odot}$, ram-pressure stripping becomes increasingly effective. However, the stripped fractions calculated using the dynamical mass profile fits are, on average, slightly greater relative to those produced by our fiducial model. This effect is evident in the far right panel of Fig. 4, which shows the difference in the stripped fraction for each object as we vary the restoring mass profile. For the dynamical fits to an NFW and Burkert profile (magenta hexagons versus cyan diamonds), we find a mean difference in $f_{\text {stripped }}$ of -0.32 and -0.15 , respectively. On average, the dynamical fits lead to greater stripped fractions, consistent with these objects being hosted by less-concentrated (or lower-mass) dark matter haloes at fixed stellar mass. While there is not perfect agreement between the different halo mass estimators, by adopting the SMHM relation as our fiducial method, we are likely underestimating the stripped fraction 

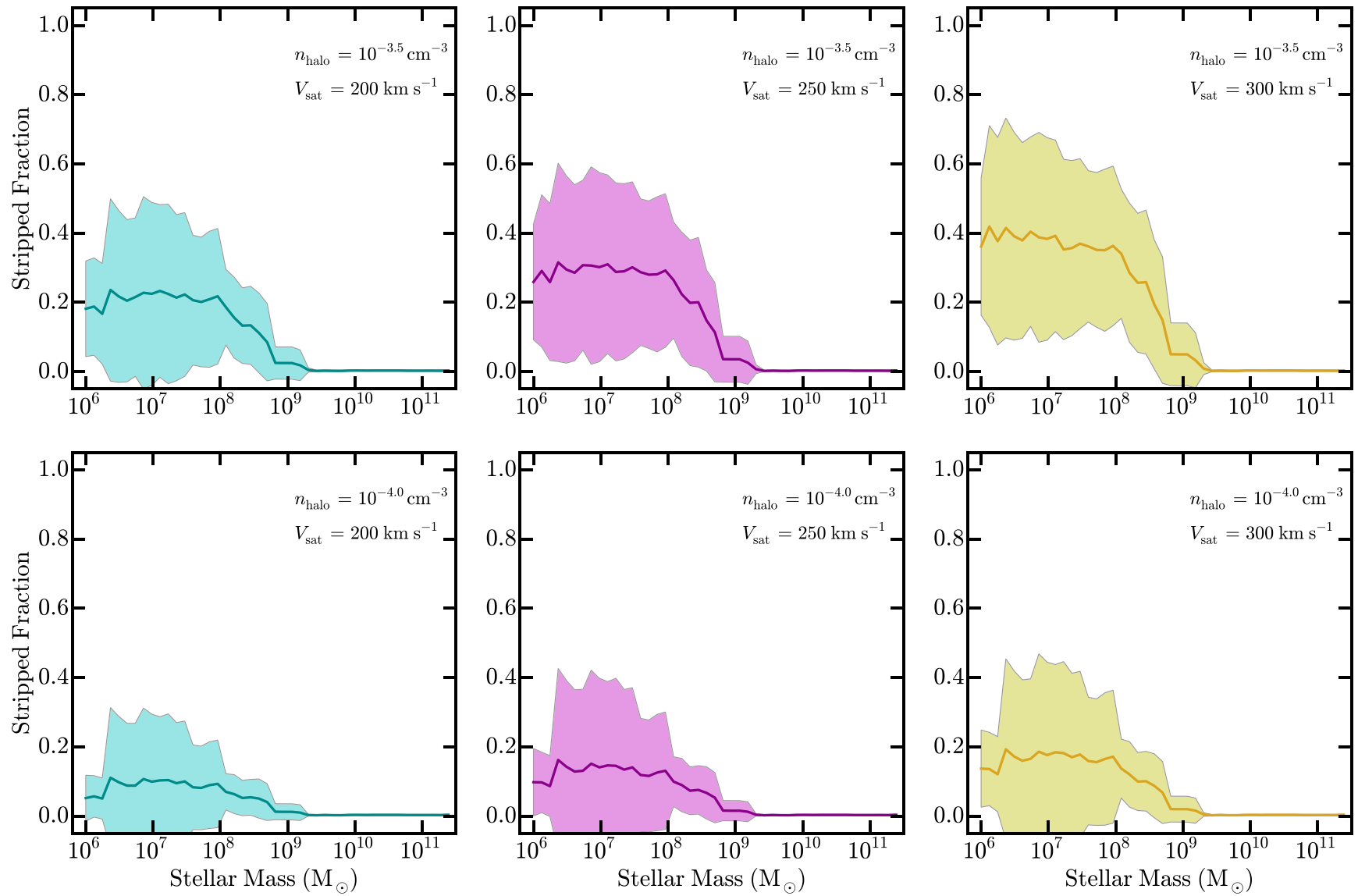

Figure 5. The fraction of $\mathrm{H}_{\mathrm{I}}$ gas stripped ( $\left.f_{\text {stripped }}\right)$ via ram-pressure stripping as a function of satellite stellar mass for our sample of $66 \mathrm{dwarf}$ galaxies. The solid line in each panel gives the mean $f_{\text {stripped }}$ in a sliding bin of width 0.6 dex in stellar mass, with the shaded region tracing the corresponding $1 \sigma$ scatter In the top and bottom rows, we assume a host halo gas density of $n_{\text {halo }}=10^{-3.5} \mathrm{~cm}^{-3}$ and $10^{-4.0} \mathrm{~cm}^{-3}$, respectively. From left to right, the satellite velocity varies from 200 (cyan) to 250 (magenta) to $300 \mathrm{~km} \mathrm{~s}^{-1}$ (gold). While the efficiency of ram-pressure stripping depends on the assumed properties of the host halo, such that $<f_{\text {stripped }}>$ ranges from $\sim 10-40$ per cent, the satellite stellar mass where ram-pressure stripping becomes significant is universally $<10^{9} \mathrm{M}_{\odot}$.

and thus providing a conservative estimate of the effectiveness of ram-pressure stripping.

\subsection{Dependence on $n_{\text {halo }}$ and $V_{\text {sat }}$}

In addition to the uncertainty in the restoring mass profile, the amount of cold gas stripped from each dwarf is highly dependent on the properties of the host system (i.e. $n_{\text {halo }}$ and $V_{\text {sat }}$ ). The density of the host gas halo, for the Local Group in particular, is relatively poorly constrained. To explore how variation in these global parameters will impact our results, we measure the stripped fraction for our sample while varying both the satellite velocity, $V_{\text {sat }}=\{200$, $250,300\} \mathrm{km} \mathrm{s}^{-1}$, and the density of the halo gas, $n_{\text {halo }}=\left\{10^{-4.0}\right.$, $\left.10^{-3.5}\right\} \mathrm{cm}^{-3}$.

Fig. 5 shows the mean and $1 \sigma$ scatter in the stripped fraction as a function of satellite stellar mass for the adopted variation in both the density of the CGM and the satellite velocity relative to the frame of reference of the host. Across the entire range of $V_{\text {sat }}$ and $n_{\text {halo }}$ explored, ram-pressure stripping becomes effective at roughly the same critical mass scale $\left(10^{8-9} \mathrm{M}_{\odot}\right)$. The efficacy of ram-pressure stripping at low masses, however, is highly dependent on the chosen parameters for $n_{\text {halo }}$ and $V_{\text {sat }}$. For example, at $M_{\star}<$ $10^{9} \mathrm{M}_{\odot}$, the stripped fraction decreases, on average, by $\sim 0.15$ as the satellite velocity is reduced from 300 to $200 \mathrm{~km} \mathrm{~s}^{-1}$ at fixed $n_{\text {halo }}$. Similarly, decreasing the host halo density from $n_{\text {halo }}=10^{-3.5} \mathrm{~cm}^{-3}$ to $10^{-4} \mathrm{~cm}^{-3}$ yields an average reduction in $f_{\text {stripped }}$ of $\sim 0.2$ at fixed $V_{\text {sat }}$. The scatter in $f_{\text {stripped }}$ associated with variation in $V_{\text {sat }}$ is particularly noteworthy, given that in our analysis we adopt a single value of $V_{\text {sat }}$ for the entire satellite population, thereby neglecting objects that have velocities greater (or less) than this value. At pericentric passage, for example, roughly 60 per cent of subhaloes in ELVIS have velocities greater than $300 \mathrm{~km} \mathrm{~s}^{-1}$. This fraction increases to 82 percent and 96 percent for $V_{\text {sat }}$ values of 250 and $200 \mathrm{~km} \mathrm{~s}^{-1}$, respectively.

\subsection{Turbulent viscous stripping}

While ram-pressure stripping is effective at removing gas from satellites below the critical quenching mass scale $\left(\lesssim 10^{8} \mathrm{M}_{\odot}\right)$, it is only able to strip roughly half of the cold gas reservoir on average. As discussed in Section 1, however, infalling satellite systems are also subject to turbulent viscous stripping, which results from $\mathrm{K}-\mathrm{H}$ instabilities at the interface of the satellite's ISM and the CGM. To estimate the impact of this secondary stripping mechanism, we 

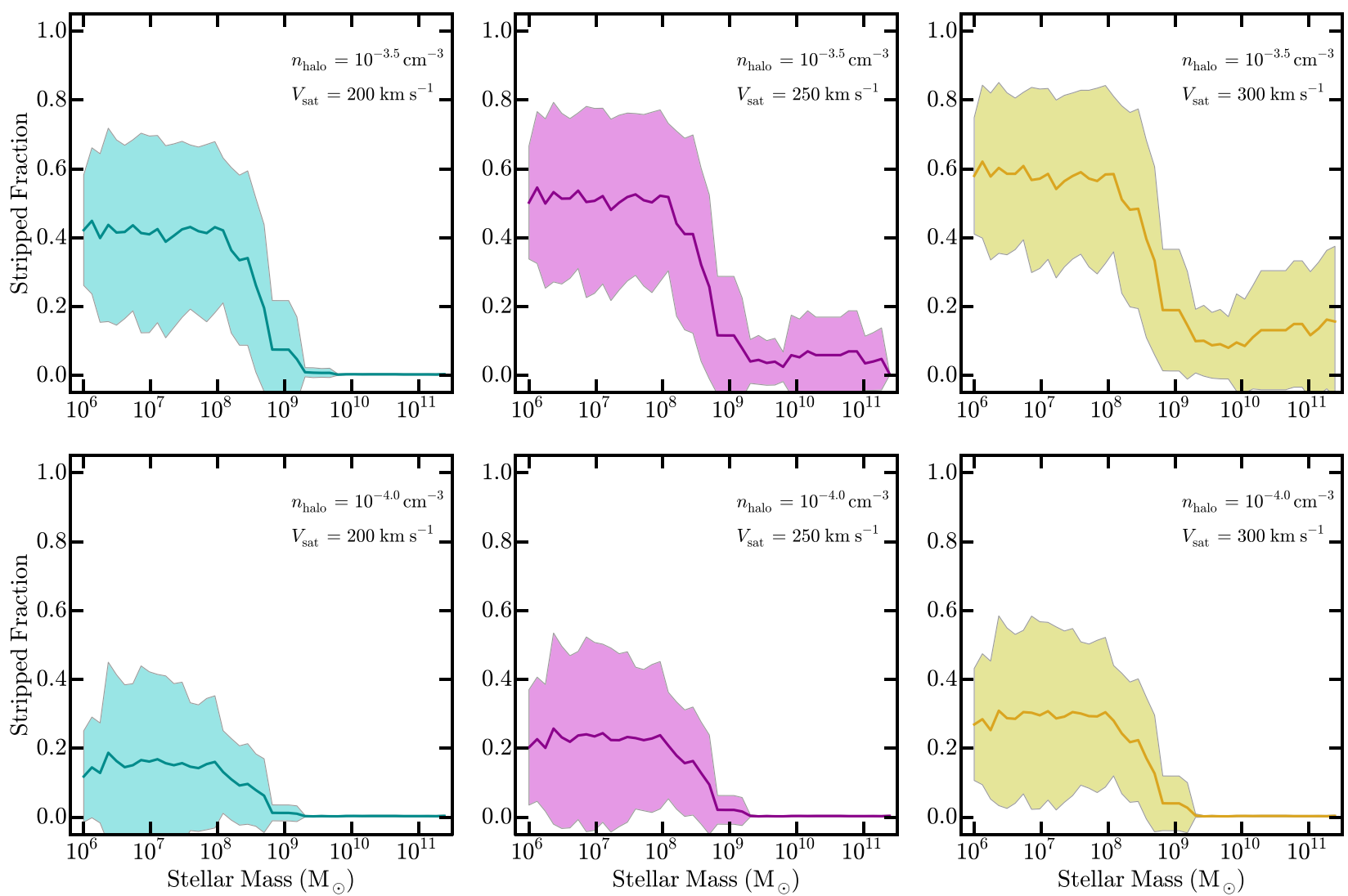

Figure 6. The fraction of $\mathrm{H}_{\mathrm{I}}$ gas stripped $\left(f_{\text {stripped }}\right)$ via ram-pressure and turbulent viscous stripping as a function of satellite stellar mass for our sample of 66 dwarf galaxies. The solid line in each panel gives the mean $f_{\text {stripped }}$ in a sliding bin of width 0.6 dex in stellar mass, with the shaded region tracing the corresponding $1 \sigma$ scatter. In the top and bottom rows, we assume a host halo gas density of $n_{\text {halo }}=10^{-3.5} \mathrm{~cm}^{-3}$ and $10^{-4.0} \mathrm{~cm}^{-3}$, respectively. From left to right, the satellite velocity varies from 200 (cyan) to 250 (magenta) to $300 \mathrm{~km} \mathrm{~s}^{-1}$ (gold). Including both ram-pressure and turbulent viscous stripping, we find an increase in the fraction of stripped gas, such that the majority of gas is removed from low-mass satellites orbiting hosts with a halo gas density of $10^{-3.5} \mathrm{~cm}^{-3}$.

allow turbulent viscous stripping to proceed for up to 1 Gyr following the initial ram-pressure stripping.

Fig. 6 shows the mean and $1 \sigma$ scatter in the stripped fraction due to both ram-pressure and turbulent viscous stripping as a function of satellite stellar mass, assuming the same range of $V_{\text {sat }}$ and $n_{\text {halo }}$ values as in Fig. 5. In contrast to when ram-pressure acts alone, the inclusion of turbulent viscous stripping at high satellite velocities $\left(V_{\text {sat }} \geq 250 \mathrm{~km} \mathrm{~s}^{-1}\right)$ and host halo densities $\left(n_{\text {halo }} \geq 10^{-3.5} \mathrm{~cm}^{-3}\right)$ yields non-zero stripped fractions for some massive satellites. As discussed in Section 4, the amount of gas removed, however, is relatively modest $\left(<f_{\text {stripped }}>\lesssim 0.2\right)$, in agreement with the observations of massive satellites in the Local Group. At low stellar mass, the efficacy of stripping is notably increased when including turbulent viscous effects, such that the typical stripped fraction is roughly $1.5 \times$ that produced by ram-pressure stripping alone. For a host halo density of $n_{\text {halo }}=10^{-3.5}$, the majority of satellites in our sample are stripped of more than half of their cold gas reservoirs.

\section{DISCUSSION}

Recent studies of satellite galaxies in the local Universe find that the efficiency of satellite (or environmental) quenching - or the time-scale upon which it occurs following infall - strongly depends on the mass of the satellite system (De Lucia et al. 2012;
Wetzel et al. 2013, 2015b; Wheeler et al. 2014; Fillingham et al. 2015). For satellites with $M_{\star} \gtrsim 10^{8} \mathrm{M}_{\odot}$, the long quenching timescales inferred from the relatively low observed satellite quenched fractions are consistent with starvation as the dominant quenching mechanism (Fillingham et al. 2015, see also Davies et al. 2016). At stellar masses below a critical mass scale of $M_{\star} \lesssim 10^{8} \mathrm{M}_{\odot}$, however, the lack of quenched systems in the field combined with the very high satellite quenched fractions observed in the Local Group require a very short quenching time-scale, consistent with a physical process that acts on roughly the dynamical time, such as ram-pressure stripping (Fillingham et al. 2015; Wetzel et al. 2015b). While stripping, and in particular ram-pressure stripping, is often thought to be a possible factor in the dearth of star-forming dwarfs in the Local Group (Einasto et al. 1974; Lin \& Faber 1983; Blitz \& Robishaw 2000), these recent results provide possible benchmarks by which to measure stripping as an active quenching mechanism. In particular, does stripping become dominant at $M_{\star} \lesssim$ $10^{8} \mathrm{M}_{\odot}$, and is it strong enough to quench low-mass satellites on a time-scale of $\sim 2$ Gyr in host systems such as the Milky Way or M31?

\subsection{Reproducing the critical mass scale for satellite quenching}

As first shown by Wheeler et al. (2014, see also Slater \& Bell 2014; Phillips et al. 2015), observations of galaxies in the Local 
Volume point towards a remarkable shift in the efficiency of satellite quenching below a satellite stellar mass of $\sim 10^{8} \mathrm{M}_{\odot}$, such that quenching at low masses proceeds relatively quickly following infall. Recent analysis of much larger samples of Milky Way-like hosts in deep photometric data sets support this picture (Phillips et al., in preparation), indicating a global critical mass scale for satellite quenching. In agreement with this picture, we find that ram pressure begins to overcome the local gravitational restoring force only in systems below a stellar mass of $\sim 10^{9} \mathrm{M}_{\odot}$. Above this mass scale, dwarfs are largely resistant to ram-pressure stripping, consistent with inefficient quenching via starvation. At low masses, however, ram-pressure stripping is able to remove (at least some of) the fuel for star formation from an infalling satellite system, thus contributing to quenching and potentially driving the change in quenching efficiency below $\sim 10^{8} \mathrm{M}_{\odot}$. Moreover, when including turbulent viscous stripping, this critical scale for satellite quenching persists, with a dramatic increase in stripping efficiency evident over the full range of $n_{\text {halo }}$ and $V_{\text {sat }}$ values explored. Altogether, our results show that stripping naturally gives rise to a critical scale for satellite quenching, consistent with observations in the local Universe.

Unlike ram pressure, we find that at high halo densities and satellite velocities turbulent viscous stripping is able to remove cold gas from some infalling satellites in the high-mass regime (i.e. $>10^{9} \mathrm{M}_{\odot}$ ). The amount of gas removed, however, is relatively modest and in broad agreement with observations of massive satellites in the Local Group. For example, there is clear evidence for stripping of the LMC and SMC, which are the only Milky Way satellites more massive than $10^{8} \mathrm{M}_{\odot}$. However, the stripped gas that comprises the Magellanic Stream and Leading Arm (Mathewson, Cleary \& Murray 1974), may result from tidal effects (e.g. Lin \& Lynden-Bell 1977; Besla et al. 2010, 2012; Guglielmo, Lewis \& Bland-Hawthorn 2014) versus ram-pressure or viscous stripping (e.g. Moore \& Davis 1994; Mastropietro et al. 2005; Hammer et al. 2015; Salem et al. 2015). Given the uncertainties associated with the orbit of the LMC and SMC and the physical origins of the stripped gas, it is difficult to make a clear accounting of the stripped fraction for the two systems (e.g. D'Onghia \& Fox 2015). However, the total H I gas mass attributed to the Magellanic Stream and Leading Arm versus that of the two Magellanic Clouds jointly is consistent with an average stripped fraction for the two systems of $\lesssim 25$ percent, consistent with our expectations at $M_{\star}>10^{8} \mathrm{M}_{\odot}$ (see Fig. 6, Brüns et al. 2005; Nidever, Majewski \& Butler Burton 2008; Nidever et al. 2010).

Finally, the mass scale at which stripping begins to be effective should depend directly on the properties of the host system (i.e. $n_{\text {halo }}$ and $V_{\text {sat }}$ in our analysis). That is, these parameters should, on average, scale with the mass of the host, such that a typical infalling satellite would experience a stronger ram pressure in more massive host systems and the critical mass scale for satellite quenching would increase accordingly. While values of $n_{\text {halo }}$ and $V_{\text {sat }}$ consistent with those expected for the Milky Way lead to an onset of stripping at roughly $\sim 10^{8-9} \mathrm{M}_{\odot}$, observations of more massive host systems, such as rich groups and clusters, should yield high quenched fractions at yet higher masses as the critical quenching mass increases with host mass. This picture is supported by observations of local clusters, which find very few star-forming satellites at stellar masses of $10^{9.5} \mathrm{M}_{\odot}$ versus the $\sim 30$ per cent quenched fraction measured for group and Milky Way-like hosts (e.g. Smith et al. 2012; Boselli et al. 2014b; Phillips et al. 2015; Sánchez-Janssen et al. 2016).

\subsection{Does stripping quench low-mass satellites?}

While ram-pressure stripping acts at the correct mass scales, our analysis finds that ram pressure alone is unable to suppress star formation in infalling satellites on the time-scales predicted by Fillingham et al. (2015). As shown in Fig. 5, for expected values of $n_{\text {halo }}$ and $V_{\text {sat }}$, infalling satellites are typically ram-pressure stripped of $<50$ per cent of their cold gas reservoirs. Given the typical H I gas fractions and star formation rates for low-mass field dwarfs, which imply exceptionally long depletion time-scales (Skillman, Côté \& Miller 2003; Geha et al. 2006; Schiminovich et al. 2010), a satellite stripped of only 50 per cent of its cold gas will still retain enough fuel to potentially form stars for many Gyr. For $n_{\text {halo }}=10^{-3.5} \mathrm{~cm}^{-3}$ and $V_{\text {sat }}=300 \mathrm{~km} \mathrm{~s}^{-1}$, the gas fractions for our satellite population, following ram-pressure stripping, are still typically $\sim 3 \times$ greater than the current observational limits for quenched satellites in the Local Group (i.e. $f_{\mathrm{HI}} \lesssim 0.136$; Spekkens et al. 2014); that is, ram pressure only quenches $\lesssim 15$ per cent of our low-mass satellite population, such that $f_{\mathrm{HI}}<0.136$. Ultimately, to reproduce the observed H I gas fractions for satellites in the Local Group and thus the inferred satellite quenching time-scales at low stellar masses, ram-pressure stripping would need to be substantially more efficient than our predictions (i.e. stripping nearly the entire cold gas reservoir of all systems, $<f_{\text {stripped }}>\sim 0.9$ ). Emerick et al. (2016) come to a similar conclusion based on wind-tunnel modelling of an idealized Leo T-like satellite during infall. Using the FLASH hydrodynamics code (Fryxell et al. 2000), they find that ram-pressure stripping is unable to fully strip the satellite within $2 \mathrm{Gyr}$.

With the inclusion of viscous effects in our fiducial model, stripping is able to remove the majority of cold gas from the low-mass, infalling satellite population (i.e. at $M_{\star}<10^{8} \mathrm{M}_{\odot}$, see Fig. 6). In 60 percent of low-mass systems following stripping, we find $\mathrm{H}$ I gas fractions consistent with the observed limits for the Milky Way dwarf spheroidal population $\left(f_{\mathrm{HI}}<0.136\right)$, such that stripping is nearly able to reproduce the high satellite quenched fraction observed in the Local Group $\left(f_{\text {quenched }} \sim 0.9-1\right)$. For roughly 40 per cent of systems, however, the resulting gas fractions - post stripping are still greater than that observed for satellites of the Milky Way. Again, to bring our satellite population into agreement with the roughly $90-100$ per cent satellite quenched fraction at low masses in the Local Group requires yet stronger stripping, such that the typical stripped fraction is closer to $f_{\text {stripped }} \sim 0.9$ at $M_{\star} \lesssim 10^{8} \mathrm{M}_{\odot}$.

While current observations of comparable nearby systems (e.g. M81, M106) find low-mass satellite populations that roughly mirror that found in the Local Group (Kaisin \& Karachentsev 2013; Spencer, Loebman \& Yoachim 2014), it remains possible that the low-mass satellite quenched fractions for the Milky Way and M31 are abnormally high relative to comparable host haloes. As shown in fig. 3 of Fillingham et al. (2015), if the satellite quenched fraction for a Milky Way-like halo is typically $\sim 70$ percent (versus 90 100 percent), the satellite quenching time-scale increases to $\sim 4$ 5 Gyr (versus $\sim 1-2$ Gyr). In such a scenario, stripping would not need to fully quench infalling satellites; instead, stripping could simply remove roughly half of a satellite's cold gas supply, so as to decrease the depletion (i.e. starvation) time-scale accordingly. Our analysis suggests that this level of stripping is very much realistic for a Milky Way-like environment, even from ram pressure alone.

\subsection{The efficacy of stripping: refining our analysis}

Within our analysis, there are several factors or approximations by which we are likely over- or under-estimating the true effectiveness 
of stripping. For example, we assume instantaneous ram-pressure stripping, which likely overestimates the ram pressure (Tonnesen et al. 2016). Moreover, like many studies of ram-pressure stripping, we adopt a smooth host halo in equilibrium with the dark matter potential. X-ray observations, however, find that massive hot haloes, typically associated with galaxy clusters, exhibit structure on scales of $\lesssim 1$ Mpc (Buote \& Tsai 1996; Schuecker et al. 2001). In addition, quasar absorption-line studies of low- and high- $z$ hosts find significant clumpiness in the CGM of massive galaxies (e.g. Thom et al. 2012; Arrigoni Battaia et al. 2015). What the observed substructure in these systems implies for a typical Milky Way-like object is unclear, but the assumption of a smooth halo certainly ignores potentially important details. Specifically, a clumpy gas halo will yield regions of higher density and thus an increased ram pressure. Such a halo is also likely to have some net velocity relative to the host's dark matter halo (our point of reference in determining satellite velocities, see Section 2.2). As shown by Tonnesen \& Bryan (2008), bulk motion of the gas halo tends to be in the same direction as that of the satellites, leading to a smaller effective $V_{\text {sat }}$ and thus weaker ram pressure. Clearly, more detailed hydrodynamical simulations are needed to fully address the impact of a clumpy CGM on our calculations. However, to quench roughly 80-90 per cent of satellites in our sample via stripping (i.e. including ram-pressure and viscous effects) requires local variations in the halo gas density on the order of $2-3 \times$ that assumed in our fiducial model (i.e. $n_{\text {halo }}$ $\left.=10^{-3.25}-10^{-3} \mathrm{~cm}^{-3}\right)$, given a satellite velocity of $300 \mathrm{~km} \mathrm{~s}^{-1}$. As shown in Fig. 7, by including a clumpy CGM, our analysis is able to reproduce the observed $\mathrm{H}_{\mathrm{I}}$ gas fractions for the Local Group satellite population, with $\sim 90$ per cent of our satellite population quenched following infall. To achieve the same quenched efficiency with a satellite velocity of only $200 \mathrm{~km} \mathrm{~s}^{-1}$, thereby accounting for potential bulk motion of the halo gas, our analysis requires a local increase in CGM density of roughly $10-20 \times$ that of our fiducial model (i.e. $n_{\text {halo }}=10^{-2.5}-10^{-2.2} \mathrm{~cm}^{-3}$ ). While such extreme CGM densities are unrealistic on average, local variations of this scale are in good agreement with the results of recent hydrodynamic simulations of stripping by Bahé \& McCarthy (2015), which find that galaxies undergoing stripping in groups and clusters typically experience increased ram pressure associated with CGM overdensities as large as $100 \times$ the mean.

Along with potential variations in the density of the host CGM, the efficacy of stripping is also impacted by the density of the satellite dark matter halo. As discussed in Section 3.1, the satellite dark matter halo mass profiles assumed in our fiducial model yield a potentially significant underestimate of the stripping efficiency. If low-mass dwarfs live in less-concentrated host haloes, as suggested by their observed internal kinematics, the resulting stripped fractions should increase by $\gtrsim 10$ per cent. With the inclusion of cored mass profiles, stripping becomes an increasingly realistic physical driver for the high quenched fractions and short quenching timescales for low-mass satellites.

While adopting less-concentrated mass profiles alone will likely not be enough to fully strip all systems, it emphasizes another missing ingredient in our analysis: baryonic feedback. As shown by Geha et al. (2012), field dwarfs (and thus infalling satellites) are nearly universally star-forming at stellar masses of $\lesssim 10^{9} \mathrm{M}_{\odot}$ and will be subject to the stellar feedback associated with radiation pressure and supernovae (e.g Murray, Quataert \& Thompson 2005; Hopkins et al. 2014). As part of our analysis, we employ observed $\mathrm{H}$ I surface density profiles for a sample of star-forming field dwarfs, along with halo masses modelled from spatially resolved kinematics, which should thus naturally capture the impact of feedback

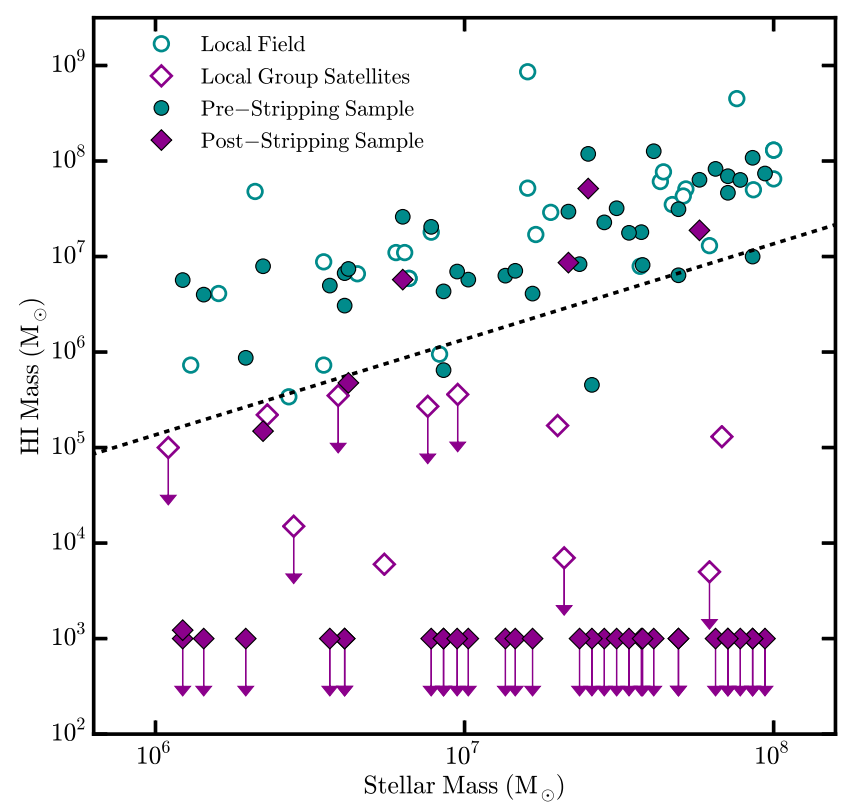

Figure 7. The $\mathrm{H}_{\mathrm{I}}$ gas mass as a function of stellar mass for our galaxy sample at $M_{\star}<10^{8} \mathrm{M}_{\odot}$, prior to infall (filled cyan circles) and after interaction with the host CGM (filled magenta diamonds). To account for potential variations in the local halo gas density, we assume $n_{\text {halo }}=10^{-3} \mathrm{~cm}^{-3}$ and $V_{\text {sat }}$ $=300 \mathrm{~km} \mathrm{~s}^{-1}$, including both ram pressure and turbulent viscous effects in our stripping calculations. For comparison, we show the observed $\mathrm{H}$ I gas masses for field galaxies in the Local Volume (open cyan circles) and satellites of the Local Group (open magenta diamonds) from McConnachie (2012) and Spekkens et al. (2014). Arrows indicate observed upper-limits or systems that are completely stripped in our analysis. The dashed black line corresponds to an $\mathrm{H}_{\mathrm{I}}$ gas fraction of 0.136 , below which we define a galaxy as quenched. Including a clumpy host CGM, we find that stripping is able to quench $\sim 90$ per cent of our infalling satellite population at low masses (33 out of 37 systems).

on $\Sigma_{\text {gas }}$ and $M(r)$. Infalling satellites, however, may experience an elevated level of star formation and thus feedback. This increased star formation activity would result from compression of the satellite's ISM due to interaction with the host CGM, thereby increasing the local gas density and allowing star formation to proceed at an accelerated rate. For example, simulations of massive satellites in groups and clusters find that ram pressure often leads to a burst of star formation for the infalling system (Fujita \& Nagashima 1999; Bekki \& Couch 2003; Bekki 2014). Within the Local Group, modelling of the orbit and star formation history of Leo I suggest that it experienced a small burst of star formation at pericentric passage prior to being quenched, consistent with being initiated by ram pressure (Boylan-Kolchin et al. 2013; Sohn et al. 2013; Weisz et al. 2014). This increase in star formation, driven by interaction with the host CGM, can inject energy into the ISM of the satellite, puffing up the system and thereby making it more susceptible to stripping (Stinson et al. 2007; El-Badry et al. 2016, but see also Emerick et al. 2016). High-resolution hydrodynamic simulations of Milky Way- or Local Group-like environments should provide a robust means for studying the possible importance of feedback in increasing the efficiency of stripping (e.g. Zolotov et al. 2012; Mistani et al. 2016; Wetzel et al. 2016; Garrison-Kimmel et al., in preparation). 


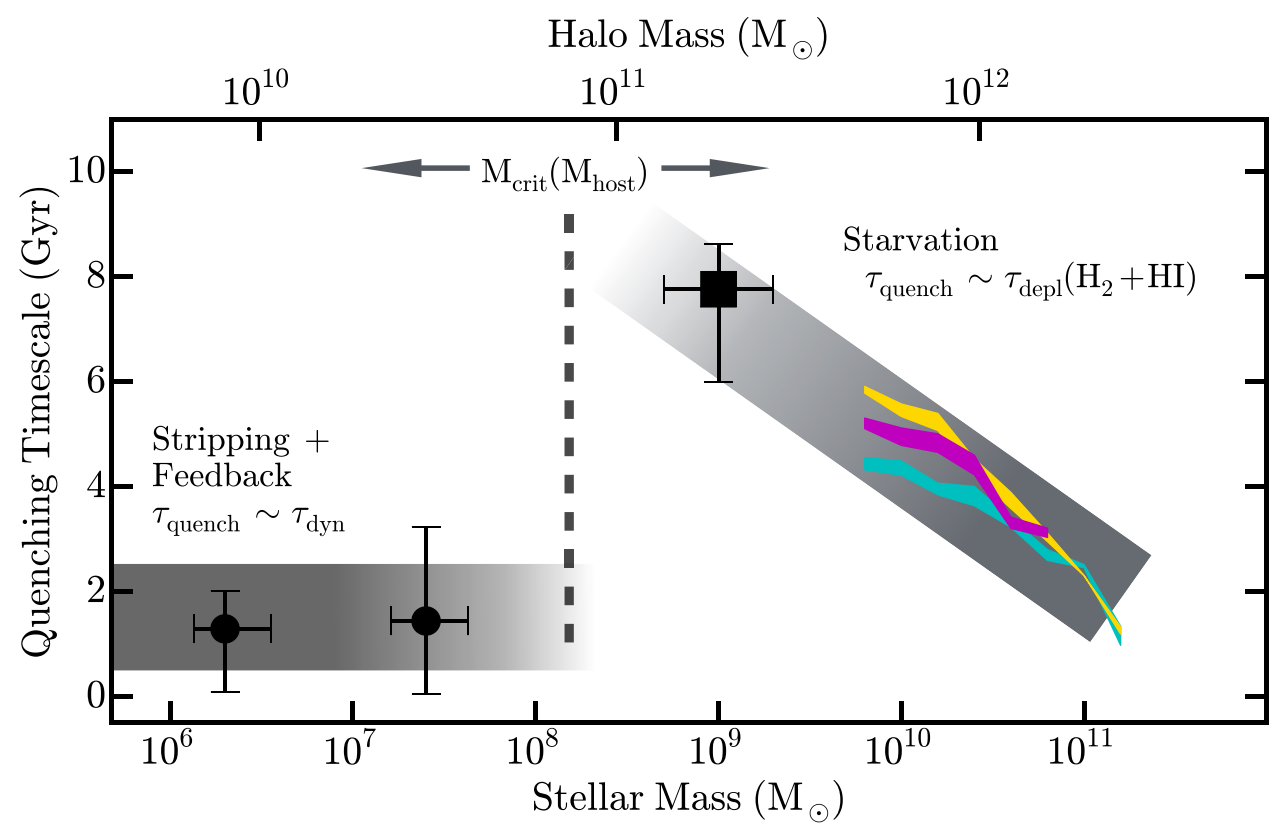

Figure 8. The dependence of the satellite quenching time-scale on satellite stellar mass in Milky Way-like and more massive host haloes (>10 $\left.0^{12} \mathrm{M}_{\odot}\right)$, as adapted from Fillingham et al. (2015). The magenta, gold, and cyan colored bands show the constraints from Wetzel et al. (2013) for satellites in host haloes of $M_{\text {host }} \sim 10^{12-13} \mathrm{M}_{\odot}, 10^{13-14} \mathrm{M}_{\odot}, 10^{14-15} \mathrm{M}_{\odot}$, respectively. The black square and circles correspond to the typical quenching time-scale for intermediateand low-mass satellites from Wheeler et al. (2014) and Fillingham et al. (2015), respectively. The light grey shaded regions highlight the expected dominant quenching mechanism as a function of satellite mass, while the vertical dashed black line denotes the critical mass scale below which satellite quenching becomes increasingly efficient. At $M_{\star} \gtrsim 10^{8} \mathrm{M}_{\odot}$, the satellite quenching time-scales show broad agreement with the observed gas depletion time-scales for field systems, suggesting that starvation is the main driver of satellite quenching at these masses. At low masses, stripping - potentially assisted by stellar feedback and a clumpy host CGM - is the most probable mechanism responsible for the high satellite quenched fractions and short quenching time-scales observed in the Local Group. The critical satellite stellar mass, $M_{\text {crit }}$, at which the dominant quenching mechanism shifts from starvation to stripping should depend on the halo mass of the host system, with more massive hosts able to strip more massive satellites.

\subsection{Towards a complete picture of satellite quenching}

Fig. 8 presents current constraints on the satellite quenching timescale from Wetzel et al. (2013), Wheeler et al. (2014), and Fillingham et al. (2015) along with a qualitative depiction of the dominant quenching mechanisms likely at play as a function of satellite stellar mass. As illustrated by Fillingham et al. (2015), the measured satellite quenching time-scales at high masses, including the lack of strong dependence on host halo mass, are broadly consistent with the expectations for quenching via starvation (see also van den Bosch et al. 2008; Wetzel et al. 2013). At low masses, on the other hand, the short quenching time-scales inferred from analysis of the Local Group satellite population are difficult to fully explain. As our analysis shows, stripping is a likely culprit in suppressing star formation at low masses, as it qualitatively reproduces the critical mass scale for satellite quenching $\left(M_{\text {crit }}\right)$ within the Local Group. However, our analysis suggests that stripping (and specifically rampressure stripping) may require a significantly clumpy host CGM or the assistance of baryonic feedback to effectively remove enough cold gas from the most gas-rich and concentrated systems. Additionally, recent work by Pearson et al. (2016) and Marasco et al. (2016) shows that the distribution of $\mathrm{H}$ I in low-mass field galaxies can be significantly altered via close encounters with neighbouring dwarfs. If these encounters occur just before or during infall on to a host system, the resulting satellite's ISM will likely be more susceptible to stripping.

If stripping drives quenching at low masses, then there are clear implications regarding the CGM of the Milky Way and similar systems. In particular, to quench all low-mass satellites within $\sim 2 \mathrm{Gyr}$ of infall requires that the CGM extends to roughly $0.5 R_{\text {vir }}$ (or $\sim 150 \mathrm{kpc}$, Fillingham et al. 2015) at a density of $n_{\text {halo }} \gtrsim 10^{-3.5} \mathrm{~cm}^{-3}$. This large physical extent is needed to explain the quenching of satellites with more circular or non-plunging orbits. In ELVIS, we find that $\sim 25$ per cent of subhaloes in our selected mass range and accreted at $0.15<z_{\text {infall }}<3$ reach their first pericentric passage at $0.5<R / R_{\text {vir }}<1$. To reproduce the extremely high satellite quenched fractions at $\lesssim 10^{8} \mathrm{M}_{\odot}$ via stripping, the CGM must therefore have a relatively cored density profile (e.g. Maller \& Bullock 2004).

While the physical picture presented in Fig. 8 broadly explains the suppression of star formation in satellite systems, it largely ignores any corresponding structural evolution. Recent observations at intermediate redshift suggest that quenching of massive central galaxies is closely associated with development of a bulge-dominated morphology (Bell et al. 2012; Cheung et al. 2012). Moreover, observations of galaxy morphology in field and group/cluster populations at $M_{\star} \gtrsim 10^{9.5} \mathrm{M}_{\odot}$ point towards an evolution of satellite systems from disc- to bulge-dominated (van den Bosch et al. 2008; Weinmann et al. 2009), suggesting that an additional mechanism beyond starvation must likely be driving satellite evolution - unless fading of the stellar population, post quenching, can account for observed differences in the light profiles of field and satellite populations. In lower-mass hosts such as the Milky Way, however, there is little morphological difference between massive satellites and field systems of comparable mass (Phillips et al. 2014). In addition, at lower satellite masses, structural evolution of satellites is relatively modest following infall and is potentially driven by baryonic feedback effects (Wheeler et al. 2015; Sánchez-Janssen et al. 2016). 


\section{SUMMARY}

Through the utilization of observed H I surface density profiles for nearby dwarf galaxies, we investigated the effectiveness of rampressure and turbulent viscous stripping in Milky Way-like environments. Our analysis was motivated by recent results which point towards a sharp change in the satellite quenching timescale, and therefore the dominant quenching mechanism, for lowmass satellite galaxies. The principal results of our analysis are as follows.

(i) Ram-pressure and turbulent viscous stripping become increasingly effective in satellite galaxies with $M_{\star} \lesssim 10^{9} \mathrm{M}_{\odot}$, consistent with the observed decrease in the satellite quenching time-scale at $M_{\star} \sim 10^{8} \mathrm{M}_{\odot}$. If stripping dominates the quenching of low-mass satellites, then we predict that the critical mass scale for satellite quenching should increase with host halo mass.

(ii) Assuming a smooth host halo with a density of $n_{\text {halo }}=$ $10^{-3.5} \mathrm{~cm}^{-3}$ and a satellite velocity of $V_{\text {sat }} \sim 300 \mathrm{~km} \mathrm{~s}^{-1}$, we find that stripping is able to remove enough cold gas so as to quench $\sim 60$ per cent of infalling satellites at low masses. However, when including a clumpy halo, such that the typical CGM density at which stripping occurs is $n_{\text {halo }} \sim 10^{-3.25}-10^{-2.5} \mathrm{~cm}^{-3}$ (i.e. $\sim 2-$ 20 times the mean density), stripping is able to effectively quench $\sim 90$ percent of infalling satellites, such that their gas fractions agree with observational limits for dwarf spheroidals in the Local Group.

(iii) The efficiency of stripping may be further enhanced with the inclusion of stellar feedback, which could play an important role in making satellite systems susceptible to ram pressure and turbulent viscous effects. Further studies of stripping via hydrodynamic simulations will be a critical step in further constraining the role of stripping in the quenching of low-mass satellites.

\section{ACKNOWLEDGEMENTS}

We thank Stephanie Tonnesen, Adam Leroy, Fabian Walter, Manoj Kaplinghat, David Buote, and David R. Jones for helpful discussions regarding this work. We also thank Deidre Hunter and John Cannon for providing $\mathrm{H}$ I data critical to the analysis. Additionally, we thank the referee for providing helpful comments which have improved the clarity of this work. This work was supported in part by NSF grants AST-1518257, AST-1517226, AST-1009973, and AST-1009999. Support for ABP was provided by a GAANN fellowship. Support for SGK was provided by NASA through Einstein Postdoctoral Fellowship grant number PF5-160136 awarded by the Chandra X-ray Center, which is operated by the Smithsonian Astrophysical Observatory for NASA under contract NAS8-03060. Support this work was provided by NASA through grants (AR-12836, AR-13896 and AR-13888) from the Space Telescope Science Institute, which is operated by the Association of Universities for Research in Astronomy, Inc., under NASA contract NAS 5-26555. MCC thanks the International Space Science Institute (ISSI) for support of this work.

This research made use of ASTROPY, a community-developed core PYTHON package for Astronomy (Astropy Collaboration et al. 2013). Additionally, the PYTHON packages NUMPY (Walt, Colbert \& Varoquaux 2011), IPYTHON (Pérez \& Granger 2007), SCIPY (Jones et al. 2001), and MATPLOTLIB (Hunter 2007) were utilized for the majority of our data analysis and presentation. Finally, we thank Robert Van Winkle for his help in collaborating and listening.

\section{REFERENCES}

Anderson M. E., Bregman J. N., 2010, ApJ, 714, 320

Arrigoni Battaia F., Hennawi J. F., Prochaska J. X., Cantalupo S., 2015, ApJ, 809,163

Astropy Collaboration et al., 2013, A\&A, 558, A33

Bahé Y. M., McCarthy I. G., 2015, MNRAS, 447, 969

Balogh M. L., Baldry I. K., Nichol R., Miller C., Bower R., Glazebrook K., 2004, ApJ, 615, L101

Bekki K., 2014, MNRAS, 438, 444

Bekki K., Couch W. J., 2003, ApJ, 596, L13

Bell E. F. et al., 2012, ApJ, 753, 167

Besla G., Kallivayalil N., Hernquist L., van der Marel R. P., Cox T. J., Kerě̌ D., 2010, ApJ, 721, L97

Besla G., Kallivayalil N., Hernquist L., van der Marel R. P., Cox T. J., Kerě̌ D., 2012, MNRAS, 421, 2109

Blitz L., Robishaw T., 2000, ApJ, 541, 675

Boselli A., Cortese L., Boquien M., Boissier S., Catinella B., Gavazzi G., Lagos C., Saintonge A., 2014a, A\&A, 564, A67

Boselli A. et al., 2014b, A\&A, 570, A69

Boylan-Kolchin M., Bullock J. S., Kaplinghat M., 2011, MNRAS, 415, L40 Boylan-Kolchin M., Bullock J. S., Kaplinghat M., 2012, MNRAS, 422, 1203 Boylan-Kolchin M., Bullock J. S., Sohn S. T., Besla G., van der Marel R. P., 2013, ApJ, 768, 140

Brüns C. et al., 2005, A\&A, 432, 45

Buote D. A., Tsai J. C., 1996, ApJ, 458, 27

Burkert A., 1995, ApJ, 447, L25

Cannon J. M. et al., 2011, ApJ, 739, L22

Cheung E. et al., 2012, ApJ, 760, 131

D’Onghia E., Fox A. J., 2015, preprint (arXiv:e-prints)

Davies L. J. M. et al., 2016, MNRAS, 455, 4013

de Blok W. J. G., McGaugh S. S., Bosma A., Rubin V. C., 2001, ApJ, 552, L23

de Blok W. J. G., Walter F., Brinks E., Trachternach C., Oh S.-H., Kennicutt R. C., Jr2008, AJ, 136, 2648

De Lucia G., Weinmann S., Poggianti B. M., Aragón-Salamanca A., Zaritsky D., 2012, MNRAS, 423, 1277

Ebeling H., Stephenson L. N., Edge A. C., 2014, ApJ, 781, L40

Einasto J., Saar E., Kaasik A., Chernin A. D., 1974, Nature, 252, 111

El-Badry K., Wetzel A., Geha M., Hopkins P. F., Kereš D., Chan T. K., Faucher-Giguère C.-A., 2016, ApJ, 820, 131

Emerick A., Mac Low M.-M., Grcevich J., Gatto A., 2016, ApJ, 826, 148

Faerman Y., Sternberg A., McKee C. F., 2016, preprint (arXiv:e-prints)

Fang T., Bullock J., Boylan-Kolchin M., 2013, ApJ, 762, 20

Fillingham S. P., Cooper M. C., Wheeler C., Garrison-Kimmel S., BoylanKolchin M., Bullock J. S., 2015, MNRAS, 454, 2039

Fox A. J., Wakker B. P., Savage B. D., Tripp T. M., Sembach K. R., BlandHawthorn J., 2005, ApJ, 630, 332

Fryxell B. et al., 2000, ApJS, 131, 273

Fujita Y., Nagashima M., 1999, ApJ, 516, 619

Garrison-Kimmel S., Boylan-Kolchin M., Bullock J. S., Lee K., 2014a, MNRAS, 438, 2578

Garrison-Kimmel S., Bullock J. S., Boylan-Kolchin M., Bardwell E., 2016, preprint (arXiv:e-prints)

Geha M., Blanton M. R., Masjedi M., West A. A., 2006, ApJ, 653, 240

Geha M., Blanton M. R., Yan R., Tinker J. L., 2012, ApJ, 757, 85

Grcevich J., Putman M. E., 2009, ApJ, 696, 385

Guglielmo M., Lewis G. F., Bland-Hawthorn J., 2014, MNRAS, 444, 1759

Gunn J. E., Gott J. R., III, 1972, ApJ, 176, 1

Gupta A., Mathur S., Krongold Y., Nicastro F., Galeazzi M., 2012, ApJ, 756, L8

Hammer F., Yang Y. B., Flores H., Puech M., Fouquet S., 2015, ApJ, 813, 110

Haurberg N. C., Salzer J. J., Cannon J. M., Marshall M. V., 2015, ApJ, 800, 121

Hester J. A., 2006, ApJ, 647, 910

Hirschmann M., De Lucia G., Wilman D., Weinmann S., Iovino A., Cucciati O., Zibetti S., Villalobos Á., 2014, MNRAS, 444, 2938 
Hopkins P. F., Kereš D., Oñorbe J., Faucher-Giguère C.-A., Quataert E., Murray N., Bullock J. S., 2014, MNRAS, 445, 581

Hunter J. D., 2007, Comput. Sci. Eng., 9, 90

Hunter D. A. et al., 2012, AJ, 144, 134

Jones E. et al., 2001, SciPy: Open source scientific tools for Python. Available at: http://www.scipy.org/ (accessed on 2015-08-25)

Kaisin S. S., Karachentsev I. D., 2013, Astrophysics, 56, 305

Kawata D., Mulchaey J. S., 2008, ApJ, 672, L103

Kenney J. D. P., Abramson A., Bravo-Alfaro H., 2015, AJ, 150, 59

Kimm T. et al., 2009, MNRAS, 394, 1131

Kimm T., Yi S. K., Khochfar S., 2011, ApJ, 729, 11

Klypin A. A., Trujillo-Gomez S., Primack J., 2011, ApJ, 740, 102

Larson R. B., Tinsley B. M., Caldwell C. N., 1980, ApJ, 237, 692

Lau M. W., Prochaska J. X., Hennawi J. F., 2015, preprint (arXiv:e-prints)

Lehner N., Howk J. C., Wakker B. P., 2015, ApJ, 804, 79

Leroy A. K., Walter F., Brinks E., Bigiel F., de Blok W. J. G., Madore B., Thornley M. D., 2008, AJ, 136, 2782

Lin D. N. C., Faber S. M., 1983, ApJ, 266, L21

Lin D. N. C., Lynden-Bell D., 1977, MNRAS, 181, 59

McConnachie A. W., 2012, AJ, 144, 4

McQuinn K. B. W. et al., 2015, ApJ, 802, 66

Maller A. H., Bullock J. S., 2004, MNRAS, 355, 694

Marasco A., Crain R. A., Schaye J., Bahé Y. M., van der Hulst T., Theuns T., Bower R. G., 2016, MNRAS, 461, 2630

Maraston C., 1998, MNRAS, 300, 872

Mastropietro C., Moore B., Mayer L., Wadsley J., Stadel J., 2005, MNRAS, 363,509

Mathewson D. S., Cleary M. N., Murray J. D., 1974, ApJ, 190, 291

Miller M. J., Bregman J. N., 2013, ApJ, 770, 118

Miller M. J., Bregman J. N., 2015, ApJ, 800, 14

Mistani P. A. et al., 2016, MNRAS, 455, 2323

Moore B., 1994, Nature, 370, 629

Moore B., Davis M., 1994, MNRAS, 270, 209

Mori M., Burkert A., 2000, ApJ, 538, 559

Murray S. D., White S. D. M., Blondin J. M., Lin D. N. C., 1993, ApJ, 407, 588

Murray N., Quataert E., Thompson T. A., 2005, ApJ, 618, 569

Navarro J. F., Frenk C. S., White S. D. M., 1997, ApJ, 490, 493

Nidever D. L., Majewski S. R., Butler Burton W., 2008, ApJ, 679, 432

Nidever D. L., Majewski S. R., Butler Burton W., Nigra L., 2010, ApJ, 723, 1618

Nulsen P. E. J., 1982, MNRAS, 198, 1007

Oh S.-H. et al., 2015, AJ, 149, 180

Pace A. B., 2016, preprint (arXiv:e-prints)

Pearson S. et al., 2016, MNRAS, 459, 1827

Pérez F., Granger B. E., 2007, Comput. Sci. Eng., 9, 21

Phillips J. I., Wheeler C., Boylan-Kolchin M., Bullock J. S., Cooper M. C., Tollerud E. J., 2014, MNRAS, 437, 1930

Phillips J. I., Wheeler C., Cooper M. C., Boylan-Kolchin M., Bullock J. S., Tollerud E., 2015, MNRAS, 447, 698

Popping G., Somerville R. S., Trager S. C., 2014, MNRAS, 442, 2398

Popping G., Behroozi P. S., Peeples M. S., 2015, MNRAS, 449, 477

Roediger E., Hensler G., 2005, A\&A, 433, 875

Salem M., Besla G., Bryan G., Putman M., van der Marel R. P., Tonnesen S., 2015, ApJ, 815, 77
Sánchez-Janssen R. et al., 2016, ApJ, 820, 69

Schiminovich D. et al., 2010, MNRAS, 408, 919

Schuecker P., Böhringer H., Reiprich T. H., Feretti L., 2001, A\&A, 378, 408

Skillman E. D., Côté S., Miller B. W., 2003, AJ, 125, 593

Slater C. T., Bell E. F., 2014, ApJ, 792, 141

Smith R. J., Lucey J. R., Price J., Hudson M. J., Phillipps S., 2012, MNRAS, 419, 3167

Sohn S. T., Besla G., van der Marel R. P., Boylan-Kolchin M., Majewski S. R., Bullock J. S., 2013, ApJ, 768, 139

Somerville R. S., Popping G., Trager S. C., 2015, MNRAS, 453, 4337

Spekkens K., Urbancic N., Mason B. S., Willman B., Aguirre J. E., 2014, ApJ, 795, L5

Spencer M., Loebman S., Yoachim P., 2014, ApJ, 788, 146

Stanimirović S., Dickey J. M., Krčo M., Brooks A. M., 2002, ApJ, 576, 773

Stinson G. S., Dalcanton J. J., Quinn T., Kaufmann T., Wadsley J., 2007, ApJ, 667, 170

Teich Y. G., McNichols A. T., Cannon J. M., 2015, IAU Gen. Assem., 22, 53290

Thom C. et al., 2012, ApJ, 758, L41

Tonnesen S., Bryan G. L., 2008, ApJ, 684, L9

Tonnesen S., Bryan G. L., van Gorkom J. H., 2007, ApJ, 671, 1434

Tonnesen S., Lu Y., Benson A., Peter A., Boylan-Kolchin M., Wetzel A. R., Weisz D. R., 2016, Am. Astron. Soc. Meeting Abstr., 227, 342.09

Tumlinson J. et al., 2013, ApJ, 777, 59

van den Bosch F. C., Aquino D., Yang X., Mo H. J., Pasquali A., McIntosh D. H., Weinmann S. M., Kang X., 2008, MNRAS, 387, 79

Walt S. v. d., Colbert S. C., Varoquaux G., 2011, Comput. Sci. Eng., 13

Walter F., Brinks E., de Blok W. J. G., Bigiel F., Kennicutt R. C., Jr, Thornley M. D., Leroy A., 2008, AJ, 136, 2563

Wang W., Sales L. V., Henriques B. M. B., White S. D. M., 2014, MNRAS, 442, 1363

Weiner B. J., Williams T. B., 1996, AJ, 111, 1156

Weinmann S. M., Kauffmann G., van den Bosch F. C., Pasquali A., McIntosh D. H., Mo H., Yang X., Guo Y., 2009, MNRAS, 394, 1213

Weisz D. R., Dolphin A. E., Skillman E. D., Holtzman J., Gilbert K. M., Dalcanton J. J., Williams B. F., 2014, ApJ, 789, 147

Weisz D. R., Dolphin A. E., Skillman E. D., Holtzman J., Gilbert K. M., Dalcanton J. J., Williams B. F., 2015, ApJ, 804, 136

Werk J. K. et al., 2014, ApJ, 792, 8

Wetzel A. R., Tinker J. L., Conroy C., van den Bosch F. C., 2013, MNRAS, 432,336

Wetzel A. R., Deason A. J., Garrison-Kimmel S., 2015a, ApJ, 807, 49

Wetzel A. R., Tollerud E. J., Weisz D. R., 2015b, ApJ, 808, L27

Wetzel A. R., Hopkins P. F., Kim J.-h., Faucher-Giguère C.-A., Kereš D., Quataert E., 2016, ApJ, 827, L23

Wheeler C., Phillips J. I., Cooper M. C., Boylan-Kolchin M., Bullock J. S., 2014, MNRAS, 442, 1396

Wheeler C., Pace A. B., Bullock J. S., Boylan-Kolchin M., Onorbe J., Fitts A., Hopkins P. F., Keres D., 2015, preprint (arXiv:e-prints)

York D. G. et al., 2000, AJ, 120, 1579

Zolotov A. et al., 2012, ApJ, 761, 71

This paper has been typeset from a $\mathrm{T}_{\mathrm{E}} \mathrm{X} / \mathrm{L} \mathrm{T} \mathrm{T}_{\mathrm{E}} \mathrm{X}$ file prepared by the author. 